\title{
Medium Access Control for Multi-Antenna Networks using Multi-User Coding
}

\author{
Christopher G. Shaw, Christian B. Peel, and A. Lee Swindlehurst \\ Brigham Young University \\ Electrical and Computer Engineering Dept. \\ 459 CB, Provo, UT 84602 \\ cgshaw@byu.edu, chris.peel@ieee.org, swindle@ee.byu.edu
}

\begin{abstract}
We present medium access controllers (MAC) for ad-hoc networks which include uplink and downlink multi-user physical layer models as well as multiple antennas. We note the advantages of these MACs over those in the literature, including a multi-antenna version of the CSMA/CA protocol used in the distributed coordination function (DCF) mode of the IEEE 802.11 MAC. Specifically, our MAC is among the first to explicitly consider multi-user techniques in an ad-hoc network, which results in superior rate performance. We include interference and network topology in the design of our protocols. We characterize MAC controllers in terms of transport capacity, and present numerical results which show the benefit of multi-user techniques in the physical layer.
\end{abstract}

\section{INTRODUCTION}

Medium access control (MAC) for complex wireless networks is an important area of research [1], [2], especially when including multiple antennas [3]-[5]. The transport capacity per node of wireless networks in many scenarios has been found to decrease with the number of nodes in a network [6], [7]. An important open question is how multiple antennas and multi-user coding impact this result. Specifically, can a wireless network with physical (PHY) and MAC layers optimized for multi-antenna and multi-user coding achieve constant or increasing per-node transport throughput as the number of nodes increases? We take important steps to answering this question in this paper.

ALOHA has been a focus of research since its inception in the late 1960s [8]. Recently there has been a significant increase in activity in multi-packet reception. This includes work showing that in some cases slotted ALOHA is the capacity-optimal MAC [9], though the focus is on an uplink scenario (not an arbitrary network). Many researchers have used directional antennas in ad-hoc networks (e.g. [10]). Our focus is on the use of antennas not just for directional diversity, but for throughput increase [11]. In [3] links are characterized as bottleneck and non-bottleneck links according to the amount of traffic required; the focus is on scheduling, rather than the PHY/MAC interaction. Antenna selection is used in a joint MACphysical layer coding scheme in [4]; one antenna at each transmitting node is used for transmission, and $N$ for reception. Thus $N$ streams can be successfully decoded; in [4] only one incoming stream per node is allowed, while the remaining $N-1$ degrees of freedom in decoding are used for interference cancellation.

In what follows, we present a MAC that uses multi-user transmission techniques in a multi-antenna ad-hoc network. We use uplink and downlink topologies at the physical layer, and use a request-tosend (RTS) / clear-to-send (CTS) handshake protocol to determine assignment of nodes as transmit, receive, or idle. Our MAC protocol uses a fixed contention period, followed by data transmission, and acknowledgment packets. We find a significant advantage to our

This work was supported by the U. S. Army Research Office under the Multi-University Research Initiative (MURI) grant W911NF-04-1-0224. techniques over CSMA/CA as used in IEEE 802.11 [12], and a pointto-point protocol similar to that of [4].

In Section II we present a model for our system, with a discussion on the transport capacities of uplink and downlink fading channels in Section III. A review of previous MAC protocols and issues of system design are presented in Section IV, with our new protocol presented in the following section. We conclude with simulation results and discussion.

\section{BACKGROUND}

\section{A. Network Model}

Consider a set of $K$ nodes communicating over wireless fading channels using $M_{k}$ antennas at the $k$ th node. Each node may transmit or receive, but may not do both at the same time. A link is an ordered pair of nodes $[n, m]$ indicating that node $n$ transmits data to node $m$. A link is active if the transmitting node is radiating a signal designated for the receiving node. Each receiving node collects radiated power from each transmitting node; if any signal is not intended for the receiving node, it is considered to be interference. The level of interference is determined by the fading coefficients between each transmitting and receiving node.

Let $T$ be the set of all transmitting nodes and let $H_{k, h}$ represent the $M_{k} \times M_{h}$ matrix of fading coefficients between nodes $h$ and $k$; then the received data at node $k$ is

$$
\mathbf{y}_{k}=\sum_{h \in T} \frac{1}{d_{k, h}^{\delta}} H_{k, h} \mathbf{x}_{h}+\mathbf{w}_{k} .
$$

Here $d_{k, h}$ is the distance between nodes $h$ and $k, \delta$ is the path-loss exponent, $\mathbf{x}_{h}$ is the signal transmitted from node $h$, and $\mathbf{w}_{k}$ is the receiver noise observed at node $k$. Each node has a transmit power constraint $P_{k}$. We assume that each node $n$ has full channel state information for all links of the form $[n, m]$. We assume that each link has a queue, which contains packets waiting to be transmitted on the link.

\section{B. Topology}

There are many different ways that a set of nodes can communicate; they can communicate in a pairwise manner, where each transmitting node designs its signal for only one receiving node, and each receiving node decodes only the signal intended for it, treating signals from other transmitting nodes as interference. This is illustrated in Figure 1, where three links are activated. We can also imagine links connected in multiple-access (uplink) topologies as illustrated in Figure 2; a network with broadcast (downlink) topologies is obtained by reversing the direction of the arrows in Figure 2.

A MAC partitions the set of nodes $\{1, \ldots, K\}$ into $L$ nonintersecting subsets, with $N_{l}$ nodes in the $l$ th subset. We label the $l$ th 

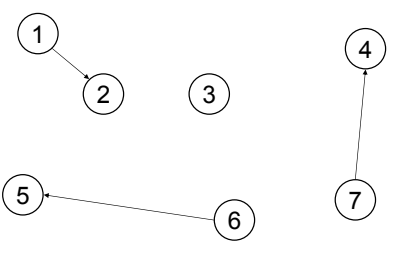

Fig. 1. A possible network link topology when only point-to-point communications links are possible.

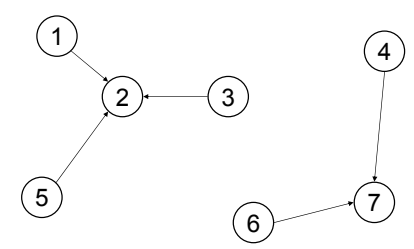

Fig. 2. Network link topology when multiple-access (uplink) communications links are possible.

subset (or sub-network) $\mathbf{z}_{l}$; nodes within $\mathbf{z}_{l}$ cooperate to exchange data, for example in an uplink topology. A node may transmit, receive, or remain idle. Signals from transmitters not in sub-network $\mathbf{z}_{l}$ cause interference at each receive node in $\mathbf{z}_{l}$. Nodes in different sub-networks are assumed to be able to exchange information when they are joined in a sub-network at a later time instant, or by the aid of a higher-layer routing protocol. Denoting the partition as $Z$, we write

$$
Z=\left\{\mathbf{z}_{l}, \ldots, \mathbf{z}_{L}\right\}
$$

We allow a partitioned sub-network to be configured as an uplink, downlink, or a point-to-point link. Figure 1 shows a network containing three point-to-point sub-networks of size $N_{l}=2$, and one sub-network containing a silent node. Figure 2 shows the same nodes configured into two sub-networks, the first of size $N_{1}=4$, and the second of size $N_{2}=3$. Other possible subset topologies that we do not have space to consider include the relay channel [13] and the interference channel [14].

Multi-hop techniques and routing are crucial for wireless network performance; to simplify our analysis, we assume that a higher layer (or protocol) provides routing between non-adjacent nodes that want to maintain data transmission. Though only single-hop transmission is considered, the transport capacity metric [6] that we maximize explicitly includes the distance that a packet must travel, as well as the number of bits communicated.

\section{CAPACITY}

We now consider several ways that a single sub-network $\mathbf{z}$ may be configured for information transfer. Figure 1 shows three pairs of nodes communicating using point-to-point links, where each receive node only knows about the corresponding transmit node, and treats other received signals as noise. The capacity-achieving transmission technique for a point-to-point network with complete channel state information (CSI) at both terminals [11] utilizes a waterfilling solution to find the power to allocate to each eigenmode of the channel. For a transmit, receive pair $[n, m]$, the received data can be modeled as

$$
\mathbf{y}_{m}=H_{m, n} \mathbf{x}_{n}+\mathbf{w}_{m} .
$$

The achievable rate is

$$
C(\mathbf{z})=\max _{\operatorname{tr}(S) \leq P_{m}} \log _{2} \frac{\left|S_{w}+H_{m, n}(\mathbf{z}) S H_{m, n}^{*}(\mathbf{z})\right|}{\left|S_{w}\right|},
$$

where $S_{w}$ is the covariance of the noise-plus-interference term $\mathbf{w}_{n}$. Though we do not show it explicitly, we emphasize that $S_{w}$ depends not only on the sub-network $\mathbf{z}$ but on other transmitting nodes in the partition $Z$. The capacity equation (4) can be solved via waterfilling to satisfy the power constraint $P_{a}$.

Transport capacity was introduced in [6] as a way to characterize the distance that information must travel as well as the quantity of information transmitted. The transport capacity for a point-to-point channel is

$$
T_{p 2 p}(\mathbf{z})=d_{m, n}(\mathbf{z}) C(\mathbf{z}) .
$$

We state the transport capacity as a function of the sub-network $\mathbf{z}$ to emphasize that the distances $d_{m, n}$, channel $H_{m, n}$, and capacity are dependent on the sub-network topology.

The second configuration of a (sub-)network we consider is in an uplink, or multiple-access topology in which many users talk with a single access point. We are interested in characterizing the uplink so that we can investigate a network that is communicating at the physical layer using several distinct multiple-access links, as shown in Figure 2. The sum capacity for the uplink channel is explored in [15].

The multi-antenna downlink has received considerable attention recently, including [16], [17]. An example of a downlink network is obtained by reversing the direction of the arrows in Figure 2. The transport capacity of the downlink channel is also explored in [15].

\section{Medium Access Control for Ad-Hoc Networks}

There are many MACs designed for wireless networks: ALOHA, slotted ALOHA, MACAW, and CSMA are all thoroughly reviewed in [18]. The algorithms of [1] adaptively minimize the power used during data transmission using intelligent decisions during the contention period. The five-phase reservation protocol [2] also includes a fixed contention window and mechanisms to tightly group data packets. These protocols are not well adapted to large ad-hoc networks and consider only single-antenna point-to-point links.

\section{A. CSMA/CA (The 802.11 DCF)}

CSMA with collision avoidance (CSMA/CA) is perhaps the most ubiquitous MAC for ad-hoc networks, due to its inclusion in the 802.11 family of standards [12] when operating in distributed coordination function (DCF) mode [18]. The physical layer of an 802.11 network typically has a fixed rate at which transmission is to occur. If the signal to interference plus noise ratio (SINR) is above the threshold $\rho$ required to achieve this rate, the transmission is successful. In a multiple-terminal link, the ability to achieve a given rate as a function of SINR is more difficult to quantify; one option is to focus solely on rate. A straightforward extension of CSMA/CA to multiple-antenna technology is to again consider transmission at a fixed rate $\gamma$, assuming a transmission is successful if the rate achievable on a link is above $\gamma$ with some small, but fixed probability of error. The rate achievable on a single point-to-point link, including receiver noise and interference from other transmitting nodes, is given by (4). 


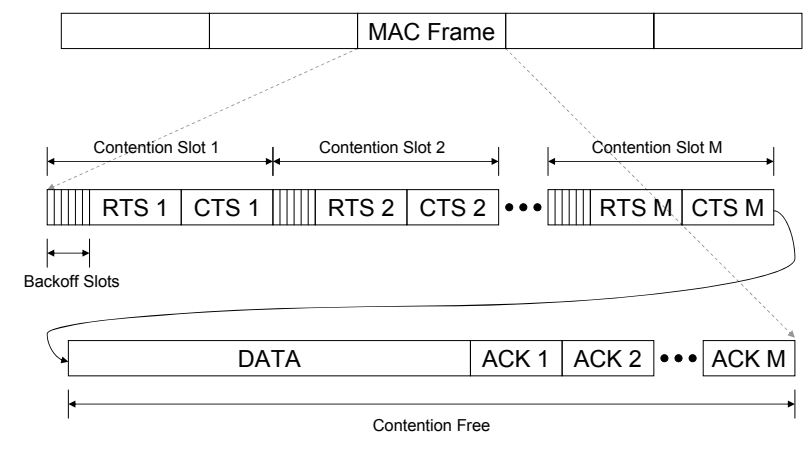

Fig. 3. Frame structure for the multi-user MAC protocol.

\section{B. MIMA-MAC}

A MAC for ad-hoc networks designed to mitigate interference using multiple antennas (MIMA) is proposed in [4] for point-to-point links. Each transmitter uses only one antenna while each receiver uses multiple antennas. MIMA-MAC uses distributed techniques to model several of these single-input multiple-output (SIMO) channels as a single MIMO channel. With appropriate channel state information (CSI), simple spatial multiplexing techniques can be used at the receiver to allow cancellation of the interfering data streams. This resolves the hidden node problem in CSMA/CA for networks with linear topologies. However, in networks with topologies where the hidden node problem is not apparent, a benefit to using MIMA-MAC over CSMA/CA is not obvious. The basic operation of MIMA-MAC includes fixed contention windows which use RTS/CTS handshakes, followed by a contention-free data window. Figure 3 illustrates this, including backoff, and acknowledgment slots.

\section{MAC FOR UPLINK AND DOWNLINK PHY LAYERS}

In contrast to the protocols presented above, we now present MAC protocols which utilize multi-user transmission and reception and multiple antennas. We use a fixed-length contention period followed by a fixed-length data period in accordance with previous research [1], [2], which found that slotted contention periods are advantageous for throughput. For the case of our protocols, where we decode or encode multiple transmissions at a node, it also makes sense to synchronize and slot these transmissions. This will ensure that idle nodes will not contend while multiple data streams are being sent and received.

We propose using multi-user coding schemes to allow each node to receive multiple data streams and thereby create uplink topologies. We expect the transport capacity of a network with downlink topologies to be less than that for a network with uplink topologies, assuming that each transmitting node (in an uplink or downlink) has the same power constraint. In a downlink scenario, the transmitting node must divide its power available between multiple transmissions. However, in an uplink, each transmitter uses the same amount of power for only one transmission. The total power input to the network is greater in the uplink scenario and the network capacity is greater. Nevertheless, we include a MAC design for downlink networks for completeness in Section V-B.

\section{A. Uplink Protocol Design}

A MAC for multi-user topologies should include two important design considerations. As noted previously, a slotted frame structure will ensure that new interferers will not contend while multiple streams are being transmitted and received. The MAC should also include a mechanism for generating the multi-user topology. Multiple RTS/CTS handshakes will allow nodes to contend and participate in uplink or downlink sub-networks. The frame structure proposed in [4] is well suited for this problem. Nodes are time synchronized and the frame is slotted. Multiple RTS/CTS handshake periods are included in the frame structure, although in [4], they are not used to create multi-user topologies.

Therefore, our uplink MAC frame structure is very similar to MIMA-MAC as illustrated in Figure 3. We include $M$ RTS/CTS handshakes, a data period, and $M$ acknowledgment slots in each MAC frame. This will ensure that there is a maximum of $M$ simultaneous data transmissions. We include multiple RTS/CTS handshakes to enable nodes to identify themselves as transmitters, receivers, and idle nodes and to organize uplink sub-networks. The basic protocol operation for our proposed uplink MAC is as follows:

RTS: At the beginning of the first RTS slot, each node with a nonzero queue will randomly choose one of $N$ backoff slots. At the beginning of the $n$th backoff slot, if a node has chosen backoff slot $n$ it will sense the channel. If the channel is free, then it will begin transmitting an RTS packet. If the channel is busy, the node will become idle for the duration of the first RTS slot. If a node received an ACK in the previous frame, it automatically chooses backoff slot $N$. In other words, a node that just successfully completed a transaction assigns itself the lowest priority for the next MAC frame. RTS slots 2 through $M$ operate similarly with two exceptions. Nodes that have received a CTS packet no longer contend. Nodes that have received other RTS packets may not transmit an RTS packet. The RTS mechanism operates similarly to MIMA-MAC with the exception that nodes that have received an RTS packet may receive more in subsequent RTS slots, but they may not transmit an RTS packet.

CTS: During each CTS slot, any node that has successfully received an RTS packet in the corresponding RTS slot sends a CTS packet. Each node may only send one CTS packet during each CTS slot but a node may send more than one CTS packet during the course of a MAC frame. This allows the organization of uplink topologies.

DATA: Nodes that have sent $k$ CTS packets receive $k$ data streams from $k$ transmitting nodes. Multi-user decoding occurs at the receiver. A transmission is successful if the rate achievable for the uplink topology (see [15]) is greater than some rate target $\gamma$. In contrast to [4] and other protocols in the literature, we use full multi-user transmission and all antennas at each user.

ACK: For each successful data transmission, the receiving node sends an ACK packet during the ACK slot corresponding to the CTS slot in which it transmitted a CTS packet. For example, if a node transmitted a CTS packet during CTS slot $k$ and successfully received the data for that transmission during the data period, then that node will send an ACK packet during ACK slot $k$.

\section{B. Downlink Protocol Design}

Our downlink MAC operates similarly to the uplink MAC. However, nodes may only send one CTS packet but multiple RTS packets per MAC frame. Multi-user encoding occurs at the transmitter. In the downlink MAC, achievable rates are obtained from expressions for downlink capacity in [15] rather than expressions for uplink capacity. Again, we use an outage criterion, and call a transmission successful if the rate $\gamma$ is supported for a particular user.

\section{Example Scenario}

Consider a network with three nodes. At the beginning of the MAC frame, node A and node B both have data to send to node C. The 


\begin{tabular}{l|r} 
Name & Value \\
\hline \# Simultaneous Links & $M$ \\
Number of Backoff Slots/Frame & 32 \\
Mini Backoff Length & $10 \mu \mathrm{sec}$ \\
RTS Length & $300 \mu \mathrm{sec}$ \\
CTS Length & $300 \mu \mathrm{sec}$ \\
ACK Length & $300 \mu \mathrm{sec}$ \\
Data Packet Length & $10 \mathrm{msec}$
\end{tabular}

TABLE I

Simulation Parameters For MiMA-MAC, Uplink, AND DOWNLINK PROTOCOLS.

distance-weighted throughput that the network achieves depends on the type of MAC used.

If we use a point-to-point MAC like CSMA/CA or MIMA-MAC, this transaction requires 2 frames. One RTS/CTS handshake will take place for the transmission from node $\mathrm{A}$ to node $\mathrm{C}$ and another for node B to node C. However, if we use the uplink MAC, the overall network throughput increases significantly. Suppose node A randomly acquires the channel before node B. During the first contention slot, node A sends an RTS to node $\mathrm{C}$ and node $\mathrm{C}$ responds with a CTS. During the second contention slot, node B sends an RTS to node C and node $\mathrm{C}$ responds with a CTS. During the data period, node A and node $\mathrm{B}$ transmit data to node $\mathrm{C}$. If the transmission is successful, node $\mathrm{C}$ responds to node $\mathrm{A}$ with an $\mathrm{ACK}$ during $\mathrm{ACK}$ slot 1 and to node $\mathrm{B}$ with an ACK during ACK slot 2.

If we use either of these point-to-point MACs, this data transaction requires at least 2 frames. When we use MIMA-MAC, the throughput is less than the throughput using CSMA/CA because time is wasted during contention slots 2 through $M$ and ACK slots 2 through $M$. However, the uplink MAC allows a multi-access topology and both data transmissions can occur simultaneously. The data transaction requires only one MAC frame.

Analysis of a simple downlink topology reveals similar results. If node $\mathrm{C}$ has data to send to nodes $\mathrm{A}$ and $\mathrm{B}$, the transaction will require two frames if either of the point-to-point MACs are used. If we use the downlink MAC, this transaction requires only one MAC frame, resulting in superior throughput performance.

\section{Simulation Results}

We implemented the MACs of Section $\mathrm{V}$ and simulated their performance using random networks. These simulation results are an upper bound on performance due to our assumption of full CSI at each transmitter. Figures 4, 5, and 6 show the performance of both the uplink and downlink MACs compared with an implementation of CSMA/CA which uses multiple antennas and a MAC similar to MIMA-MAC as proposed in [4]. In all simulations in this paper, the nodes are placed randomly on the plane, with locations chosen from a zero-mean circularly-symmetric Gaussian distribution with variance $1 / \sqrt{2}$ in each direction. The channels between each user are also chosen from a circularly-symmetric complex Gaussian with unit variance. We utilize a fixed number of antennas $M\left(M_{k}=M\right.$ for all $k$ ) at each of the $K$ nodes in the network; these antennas are used for both transmitting and receiving data, though not simultaneously.

Table I shows simulation parameters common to our implementation of MIMA-MAC and our uplink and downlink protocols. The number of simultaneous links allowed is set to be the number of antennas $M$ at each node. The number of "mini" backoff slots per frame, backoff length, length of RTS, CTS, and ACK packets and data packet length are also given. These parameters are chosen to match

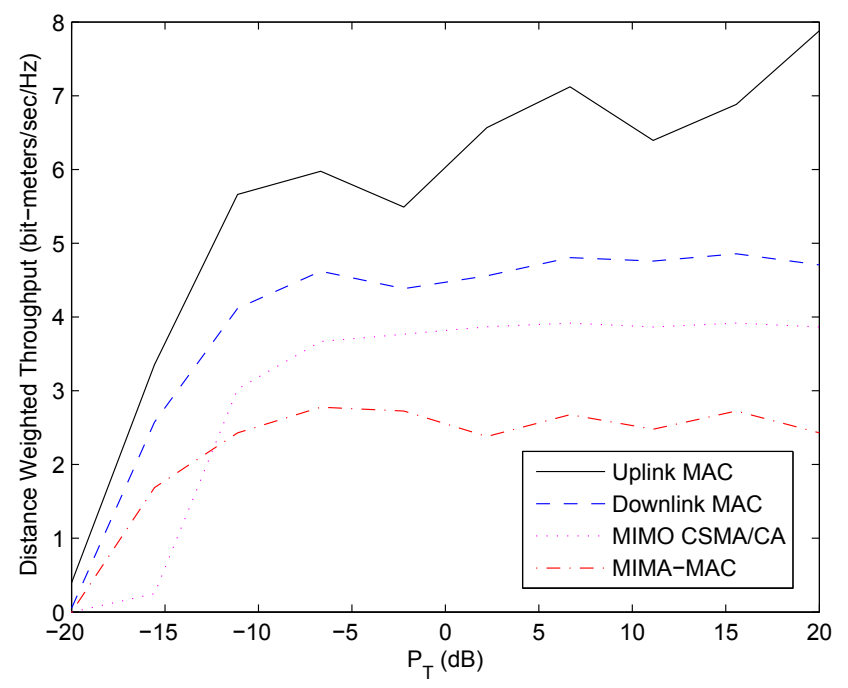

Fig. 4. The transport throughput as a function of the power constraint at each node of the random network. The uplink MAC does better than the other protocols. At low power, all protocols benefit significantly from a small increase in power, while at higher power the throughput is limited by the fixed rate target $\gamma=0.5$.

those given in [4]. The simulation parameters for our implementation of CSMA/CA are chosen to match those given in Table I.

Figure 4 shows the distance-weighted throughput of these four MACs versus the power constraint at each transmitting node $P_{k}$. We used a network with 4 nodes and 2 antennas per node. Packets are generated according to a Poisson process with mean rate $\lambda$ equal to 50 packets per second. We used 0.5 bit-meters as our rate threshold $\gamma$ and a path loss exponent of 2. At low power, all protocols benefit significantly from a small increase in power, while at higher power the throughput is limited by the fixed rate target $\gamma=0.5$. As expected, the uplink MAC achieves a higher distance-weighted throughput than the downlink MAC and both point-to-point MACs.

Figure 5 shows the distance-weighted throughput versus $\lambda$, the mean packet generation rate. Again, we used a random network with 4 nodes and 2 antennas per node. We used the same rate threshold $\gamma$ and path loss exponent. Our power constraint per transmitting node is $10 \mathrm{~dB}$. For only a few packets arriving, all protocols have similar rates, but as the rate of packet arrival increases, the uplink protocol gives a significant advantage.

Figure 6 shows the distance-weighted throughput versus $K$, the number of nodes in the network. We compare throughput as a function of the number of nodes in the network. We used three antennas per node and the same path loss exponent, mean packet generation rate, power constraint, and rate threshold as above. Although the uplink and downlink MACs decrease in capacity as more nodes are introduced, they do perform better than the other point-to-point MACs.

\section{SUMMARY}

We have presented a MAC protocol that explicitly includes multiuser uplink and downlink multi-antenna encoding and decoding at the physical layer of a wireless network. We found our multi-user technique to give a significant advantage over previous protocols, including the DCF mode of 802.11. We model the physical layer using an outage criterion to determine if the interference from other 


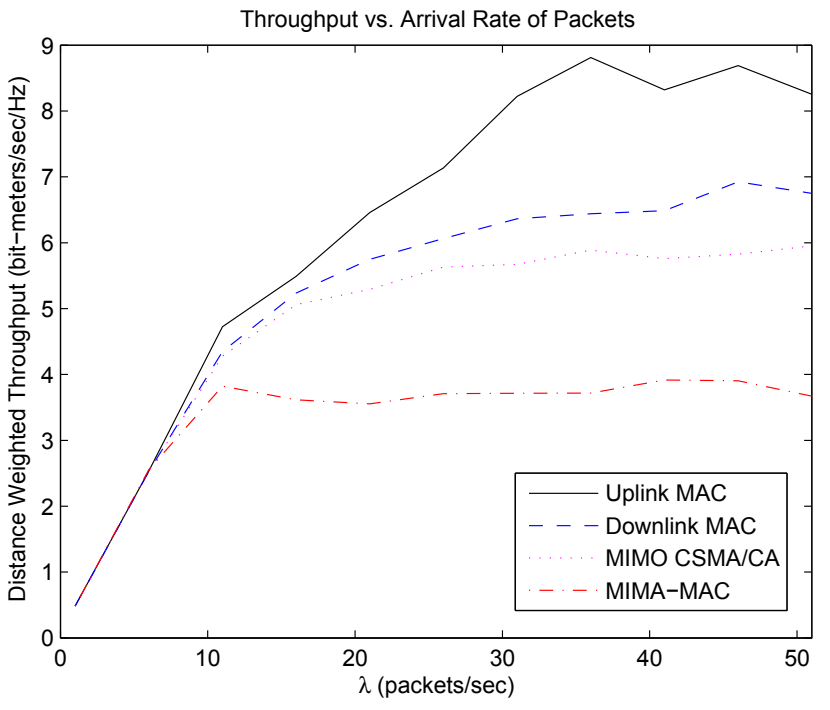

Fig. 5. The distance-weighted throughput as a function of the input rate to the random network. The uplink MAC again performs better better than the downlink MAC, CSMA/CA, or MIMA-MAC, especially for high packet arrival rates.

nodes and receiver noise allow data transmission. Distance-weighted throughput is used instead of rate as the optimization metric. The order of encoding/decoding which gives the maximum transport rate is utilized.

Future work will include the best way to obtain CSI, including appropriate inclusion of training signals and channel estimation into the MAC protocol. Our current results can be seen as an optimistic bound in that they assume that each node possesses full channel state information for channels to and from a given node. Though it is not yet clear how crucial it is to avoid interference during training, we anticipate tracking and prediction techniques will prove to be of use. Trade-offs exist between power, rate, and delay in the fading multi-user channels that we have discussed. An analytic exposition of fundamental rate-delay-power trade-offs would shed light on MAC protocol design. A related topic is the utilization of relays in the physical layer, in addition to the multi-user techniques described above. This could include the decode-and-forward and compress-andforward techniques of [13].

\section{REFERENCES}

[1] S. Toumpis and A. Goldsmith, "Performance, optimization, and crosslayer design of media access protocols for wireless ad hoc networks," in Proc. IEEE International Conference on Communications, vol. 3, (Anchorage, AK, USA), pp. 2234-2240, May 2003.

[2] C. Zhu and M. S. Corson, "A five-phase reservation protocol (fprp) for mobile ad hoc networks," Wireless Networks, vol. 7, no. 4, pp. 371-384, 2001.

[3] K. Sundaresan, R. Sivakumar, M. A. Ingram, and T.-Y. Chang, "Medium access control in ad hoc networks with MIMO links: optimization considerations and algorithms," Mobile Computing, IEEE Transactions on, vol. 3, pp. 350-365, Oct.-Dec. 2004.

[4] M. Park, R. H. Jr., and S. M. Nettles, "Improving throughput and fairness for MIMO ad hoc networks using antenna selection diversity," in Proc. Global Telecommunications Conference, vol. 5, pp. 3363-3367, IEEE, Nov.-Dec. 2004.

[5] P. Casari, M. Levorato, and M. Zorzi, "Some issues concerning mac design in ad hoc networks with mimo communications," in Proc eighth International Symposium on Wireless Personal Multimedia Communications (WPMC), (Aalborg, Denmark), September 2005.

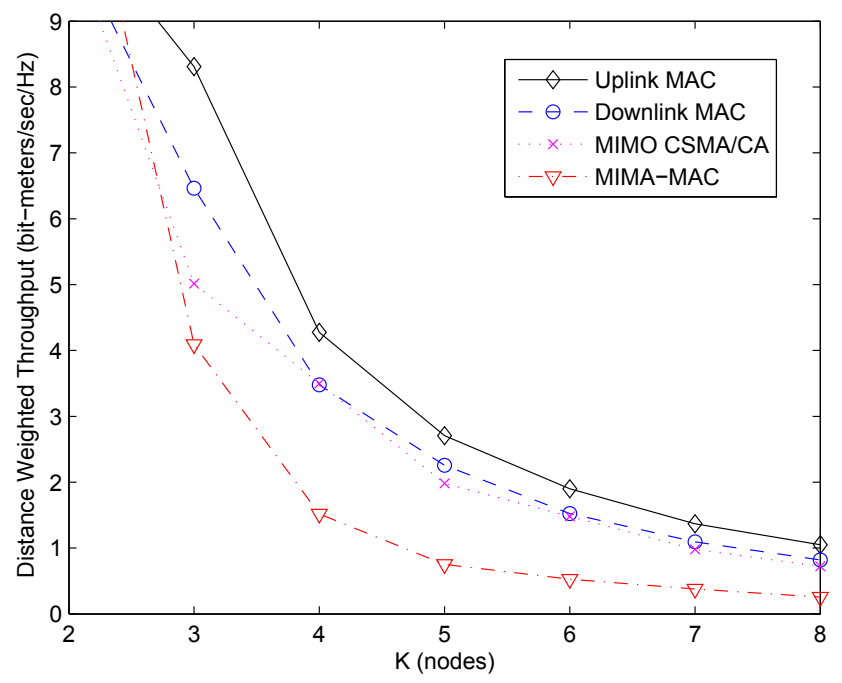

Fig. 6. The transport throughput as a function of the number of nodes in the random network. The uplink MAC performs better than the downlink or point-to-point MAC at all numbers of nodes, although the rate goes down as the number of nodes increases.

[6] P. Gupta and P. Kumar, "The capacity of wireless networks," IEEE Transactions on Information Theory, vol. 46, pp. 388-404, March 2000.

[7] E. T. Oliver Lévêque, "Information theoretic upper bounds on the capacity of large extended ad-hoc wireless networks," Submitted to the IEEE Transactions on Information Theory, 2005.

[8] N. Abramson, "Development of the ALOHANET," IEEE Transactions on Information Theory, vol. 31, pp. 119-123, March 1985.

[9] V. Naware, G. Mergen, and L. Tong, "Stability and delay of finiteuser slotted ALOHA with multi-packet reception," IEEE Transactions on Information Theory, vol. 51, pp. 2636-2656, July 2005.

[10] R. Ramanathan, J. Redi, C. Santivanez, D. Wiggins, and S. Polit, "Ad hoc networking with directional antennas: a complete system solution," Selected Areas in Communications, IEEE Journal on, vol. 23, pp. 496506, March 2005.

[11] I. E. Telatar, "Capacity of multi-antenna Gaussian channels," European Trans. Telecommunications, vol. 10, pp. 585-595, Nov/Dec 1999.

[12] "IEEE Std 802.11-1997 information technology- telecommunications and information exchange between systems-local and metropolitan area networks-specific requirements-part 11: Wireless lan medium access control (MAC) and physical layer (PHY) specifications," November 1997.

[13] G. Kramer, M. Gastpar, and P. Gupta, "Capacity theorems for wireless relay channels," in Proc. 41st Annual Allerton Conf. on Commun., Control and Comp., (Monticello, IL, USA), pp. 1074-1083, October 2003.

[14] G. Kramer, "Outer bounds on the capacity of Gaussian interference channels," IEEE Transactions on Information Theory, vol. 50, pp. 581586, March 2004.

[15] C. B. Peel, A. L. Swindlehurst, and D. Dahlhaus, "Distance-weighted throughput for multi-antenna wireless networks with multi-user links," in Vehicular Technology Conference, Spring 2006, vol. 2, pp. 901 - 905, May 2006.

[16] G. Caire and S. Shamai, "On the achievable throughput of a multiantenna Gaussian broadcast channel," IEEE Transactions on Information Theory, vol. 43, pp. 1691-1706, July 2003.

[17] H. Weingarten, Y. Steinberg, and S. Shamai, "The capacity region of the Gaussian MIMO broadcast channel," in Proceedings Conf. on Information Sciences and Systems (CISS), (Princeton, NJ), March 2004.

[18] A. S. Tanenbaum, Computer Networks. Prentice Hall PTR, fourth ed., 2003. 